\title{
DETERMINANT PRESERVING TRANSFORMATIONS ON SYMMETRIC MATRIX SPACES*
}

\author{
CHONGGUANG $\mathrm{CAO}^{\dagger}$ AND XIAOMIN TANG $^{\dagger}$
}

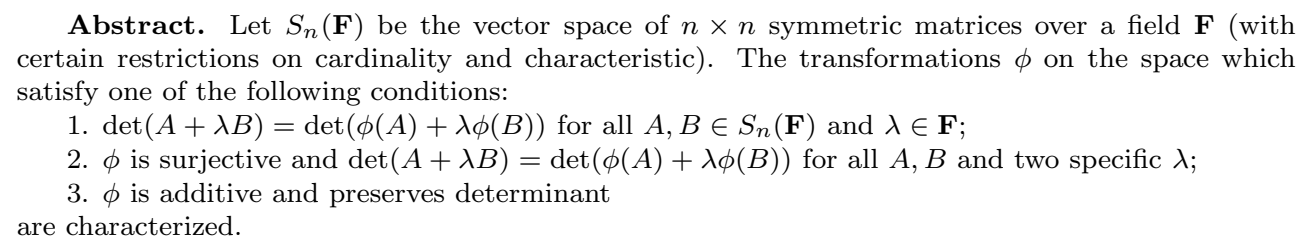

Key words. Linear preserving problem, Rank, Symmetric matrix, Determinant.

AMS subject classifications. 15A03, 15A04.

1. Introduction and statement of the results. Linear preserving problems is an active research area in matrix theory. The first result on linear preservers is due to Frobenius [1] who studied linear transformations on matrix algebras preserving the determinant. Let $M_{n}(\mathbf{F})$ be the vector space of $n \times n$ matrices over a field $\mathbf{F}$. Frobenius characterized a linear map $\phi: M_{n}(\mathbf{F}) \rightarrow M_{n}(\mathbf{F})$ satisfying

$$
\operatorname{det}(\phi(A))=\operatorname{det}(A), \quad \text { for all } A \in M_{n}(\mathbf{F})
$$

when $\mathbf{F}=\mathbb{C}$ (the complex field).

Recently, Dolinar and Šemrl [2] and Tan and Wang [4] respectively modified the problem by removing the linearity of $\phi$ and changing condition (1.1) to

$$
\operatorname{det}(\phi(A)+\lambda \phi(B))=\operatorname{det}(A+\lambda B), \quad \text { for all } A, B \in M_{n}(\mathbf{F}) \text { and all } \lambda \in \mathbf{F} \text {. }
$$

This paper is motivated by [2] and [4]. We study determinant preservers on the vector space of symmetric matrices.

Let $\mathbf{F}$ be a field. Let $\mathbf{F}^{*}$ denote the set of all nonzero elements in $\mathbf{F}$. Let $S_{n}(\mathbf{F})$ be the vector space of all $n \times n$ symmetric matrices over $\mathbf{F}$. For $A \in S_{n}(\mathbf{F})$, let $a_{i j}$ denote the $(i, j)$ - entry of $A, A^{i j}$ the $(i, j)$-cofactor of $A$, and adj $A$ the adjoint of $A$. Let $E(i, j)$ denote the matrix with 1 at the $(i, j)$ - entry and 0 elsewhere, $I_{n}$ the $n \times n$ identity matrix and $D(i, j)$ the matrix $E(i, j)+E(j, i)$ for all $i<j$. For $A \in M_{n}(\mathbf{F})$, we denote by $A^{t}$ the transpose of $A$. For $A \in S_{n}(\mathbf{F})$, we denote by $v_{A}$ the column vector

$$
\left(a_{11}, a_{12}, \cdots, a_{1 n}, a_{22}, a_{23}, \cdots, a_{2 n}, \cdots, a_{n-1, n-1}, a_{n-1, n}, a_{n n}\right)^{t} .
$$

* Received by the editors 19 February, 2004. Accepted for publication 13 September, 2004. Handling Editor: Bit-Shun Tam.

${ }^{\dagger}$ Department of Mathematics, Heilongjiang University, Harbin, 150080, P. R. China (caochongguang@163.com). Supported by the NSF of China (No. 10271021), NSF of Heilongjiang Province (No. A01-07) and the fund of Heilongjiang Education Committee for Overseas Scholars. 
We define a map $\sigma$ from $S_{n}(\mathbf{F})$ to the column space $\mathbf{F}^{\frac{n(n+1)}{2}}$ by $\sigma(A)=v_{A}$ for all $A \in S_{n}(\mathbf{F})$. Obviously, $\sigma$ is a nonsingular linear map. For $A, B \in S_{n}(\mathbf{F})$, we use $D_{A, B}(t)$ to denote the polynomial $\operatorname{det}(A+t B)$. It is easy to see that the coefficient of $t^{n-1}$ in $D_{A, B}(t)$ is equal to $\sum_{i=1}^{n} \sum_{j=1}^{n} a_{i j} B^{i j}$.

In this paper we will prove the following main results.

TheOrem 1.1. Let $\mathbf{F}$ be a field with $|\mathbf{F}| \geq n,|\mathbf{F}|>3$ and $\operatorname{ch} \mathbf{F} \neq 2$, or $\mathbf{F}=\mathbf{F}_{2}$ and $n=2$. Then $\phi: S_{n}(\mathbf{F}) \rightarrow S_{n}(\mathbf{F})$ is a transformation which satisfies the condition (1.2) $\operatorname{det}(A+\lambda B)=\operatorname{det}(\phi(A)+\lambda \phi(B))$, for all $A, B \in S_{n}(\mathbf{F})$ and all $\lambda \in \mathbf{F}$ if and only if $\phi$ has the form

$$
\phi(A)=\alpha P A P^{t}, \text { for all } A \in S_{n}(\mathbf{F})
$$

where $\alpha \in \mathbf{F}^{*}, P \in G L_{n}(\mathbf{F})$ (the set of all nonsingular matrices in $M_{n}(\mathbf{F})$ ) and $\operatorname{det}\left(\alpha P^{2}\right)=1$.

TheOrem 1.2. Let $\mathbf{F}$ be a field with $|\mathbf{F}| \geq n,|\mathbf{F}|>3$ and $\operatorname{ch} \mathbf{F} \neq 2$ and $\phi$ : $S_{n}(\mathbf{F}) \rightarrow S_{n}(\mathbf{F})$ be a surjective transformation satisfying

$$
\operatorname{det}\left(A+\lambda_{i} B\right)=\operatorname{det}\left(\phi(A)+\lambda_{i} \phi(B)\right), \quad \text { for all } A, B \in S_{n}(\mathbf{F})
$$

and $i=1,2$, where $\lambda_{i} \in \mathbf{F}^{*}$ and $\left(\lambda_{1} / \lambda_{2}\right)^{k} \neq 1$ for $1 \leq k \leq n-2$. Then $\phi$ is of the form as given by (1.3).

THeOREM 1.3. Let $\mathbf{F}$ be a field such that $\operatorname{ch} \mathbf{F}=0$ or $\operatorname{ch} \mathbf{F} \geq n$ and $|\mathbf{F}|>3$, and let $\phi: S_{n}(\mathbf{F}) \rightarrow S_{n}(\mathbf{F})$ be an additive transformation. If $\phi$ satisfies the condition $\operatorname{det} A=\operatorname{det} \phi(A)$ for all $A \in S_{n}(\mathbf{F})$, then $\phi$ is of the form as given by (1.3).

Because the proofs of Theorems 1.2 and 1.3 are similar to those for Theorems 2 and 3 of [4], they are omitted. In section 2 we will prove Theorem 1.1.

2. The proof of Theorem 1.1. In order to prove Theorem 1.1, we need several lemmas.

Lemma 2.1. [5] Suppose $\mathbf{F}$ is a field with $|\mathbf{F}|>3$, and $\phi: S_{n}(\mathbf{F}) \rightarrow S_{n}(\mathbf{F})$ is a nonsingular linear transformation which is a rank-one preserver. Then $\phi$ is of the form

$$
\phi(A)=\alpha P A P^{t} \text { for all } A \in S_{n}(\mathbf{F}),
$$

where $\alpha \in \mathbf{F}^{*}$ and $P \in G L_{n}(\mathbf{F})$.

Recall that a matrix $A \in M_{n}(\mathbf{F})$ is said to be alternate if $x^{t} A x=0$ for all $x \in \mathbf{F}^{n}$. The following result is known ([3, p.155; p.161, Theorem 7; p.171, Theorem 10]):

Remark 2.2. Let $\mathbf{F}$ be a field and let $A \in S_{n}(\mathbf{F})$. Then there exists $P \in G L_{n}(\mathbf{F})$ such that

(i) $P^{t} A P$ is a diagonal matrix if $\operatorname{ch} \mathbf{F} \neq 2$, or $\operatorname{ch} \mathbf{F}=2$ and $A$ is not alternate; and

(ii) $P^{t} A P$ is of the form $\operatorname{diag}(S, \ldots, S, 0, \ldots, 0)$, where $S=\left[\begin{array}{ll}0 & 1 \\ 1 & 0\end{array}\right]$ if $\operatorname{ch} \mathbf{F}=2$ and $A$ is alternate.

LEMma 2.3. Suppose $\phi$ is a linear transformation from $S_{n}(\mathbf{F})$ into itself satisfying $\operatorname{det} A=\operatorname{det} \phi(A)$, for all $A \in S_{n}(\mathbf{F})$. Then $\phi$ is bijective. 
Proof. It is sufficient to prove that $\phi$ is an injective transformation, or to show that $\phi(A)=0$ implies that $A=0$. Consider any $A \in S_{n}(\mathbf{F})$ such that $\phi(A)=0$. Then $\operatorname{det} A=\operatorname{det} \phi(A)=0$. This means $\operatorname{rank} A<n$. By Remark 2.2, it is readily seen that there exists $B \in S_{n}(\mathbf{F})$ such that $\operatorname{rank} B=n-\operatorname{rank} A$ and $\operatorname{det}(A+B) \neq 0$. Then we get $\operatorname{det} B=\operatorname{det} \phi(B)=\operatorname{det} \phi(A+B)=\operatorname{det}(A+B) \neq 0$. Thus we have $\operatorname{rank} B=n$. This implies $\operatorname{rank} A=0$, i.e., $A=0$.

Lemma 2.4. Suppose $\mathbf{F}$ is a field with $|\mathbf{F}|>2$ and $\operatorname{ch} \mathbf{F} \neq 2$, and $\phi$ is a linear transformation from $S_{n}(F)$ into itself satisfying $\operatorname{det} A=\operatorname{det} \phi(A)$, for all $A \in S_{n}(\mathbf{F})$. Then $\phi$ is a rank-one preserver.

Proof. We need to show that for every rank-one matrix $A \in S_{n}(\mathbf{F})$ we have $\operatorname{rank} \phi(A)=1$. By Remark 2.2, we may write $A$ as $a N E(1,1) N^{t}$ where $a \in \mathbf{F}^{*}$ and $N \in G L_{n}(\mathbf{F})$. By Lemma $2.3, \phi$ is injective; so $\phi(A) \neq 0$. If $\operatorname{rank} \phi(A)=$ $r>1$, since $\operatorname{ch} \mathbf{F} \neq 2$, by Remark 2.2 we may assume that $\phi(A)=M\left(D_{r} \oplus\right.$ $O) M^{t}$, where $M \in G L_{n}(\mathbf{F}), D_{r}=\operatorname{diag}\left(b_{1}, \cdots, b_{r}\right)$ with $b_{1}, \cdots, b_{r} \in \mathbf{F}^{*}$. Set $B=$ $M\left(\operatorname{diag}\left(0,-b_{2}, \cdots,-b_{r}\right) \oplus I_{n-r}\right) M^{t}$. By Lemma $2.3, \phi$ is surjective; so there exists $C \in S_{n}(\mathbf{F})$ such that $\phi\left(N C N^{t}\right)=B$. Hence for every $\lambda \in \mathbf{F}$, we have

$$
\begin{aligned}
& \operatorname{det}(\lambda a E(1,1)+C) \\
= & (\operatorname{det} N)^{-2} \operatorname{det}\left(N(\lambda a E(1,1)+C) N^{t}\right) \\
= & (\operatorname{det} N)^{-2} \operatorname{det}\left(\lambda A+N C N^{t}\right) \\
= & (\operatorname{det} N)^{-2} \operatorname{det}(\lambda \phi(A)+B) \\
= & (\operatorname{det} M)^{2}(\operatorname{det} N)^{-2} \operatorname{det}\left(\lambda\left(b_{1}\right) \oplus(\lambda-1) \operatorname{diag}\left(\mathrm{b}_{2}, \cdots, \mathrm{b}_{\mathrm{r}}\right) \oplus \mathrm{I}_{\mathrm{n}-\mathrm{r}}\right) .
\end{aligned}
$$

In particular, when $\lambda=0$ and 1 we obtain, respectively,

(ii) $\operatorname{det} C=(\operatorname{det} M)^{2}(\operatorname{det} N)^{-2} \operatorname{det}\left(\operatorname{diag}\left(0,-b_{2}, \cdots,-b_{r}\right) \oplus I_{n-r}\right)=0$; and

(iii) $\operatorname{det}(a E(1,1)+C)=(\operatorname{det} M)^{2}(\operatorname{det} N)^{-2} \operatorname{det}\left(\left(b_{1}\right) \oplus O_{r-1} \oplus I_{n-r}\right)=0$.

Write $C$ as $\left[\begin{array}{cc}c & w^{t} \\ w & C_{1}\end{array}\right]$, where $C_{1} \in S_{n-1}(\mathbf{F})$. By (ii) and (iii) we have

$$
a \operatorname{det} C_{1}=\operatorname{det} C+\operatorname{det}\left[\begin{array}{ll}
a & w^{t} \\
0 & C_{1}
\end{array}\right]=\operatorname{det}(a E(1,1)+C)=0 .
$$

On the other hand, since $|\mathbf{F}|>2$, we can choose $\lambda \in F, \lambda \neq 0,1$. Then by (i) and an argument similar to the above one we have

$$
\lambda a \operatorname{det} C_{1}=\operatorname{det} C+\operatorname{det}\left[\begin{array}{cc}
\lambda a & w^{t} \\
0 & C_{1}
\end{array}\right]=\operatorname{det}(\lambda a E(1,1)+C) \neq 0 .
$$

So we arrive at a contradiction. Hence $r=1$, and $\phi(A)$ is a rank-one matrix.

LEMMA 2.5. Suppose $\mathbf{F}$ is a field with $|\mathbf{F}|>3$ and $\operatorname{ch} \mathbf{F} \neq 2$. Then $\phi: S_{n}(\mathbf{F}) \rightarrow$ $S_{n}(\mathbf{F})$ is a linear transformation which satisfies $\operatorname{det} A=\operatorname{det} \phi(A)$ for all $A \in S_{n}(\mathbf{F})$, if and only if $\phi$ has the form as given by Theorem 1.1.

Proof. The "if" part is obvious. The "only if" part follows immediately from Lemmas 2.1, 2.3 and 2.4.

LEMMA 2.6. There exists a basis for $S_{n}(\mathbf{F})$ consisting of nonsingular matrices.

Proof. Denote by $W$ the set of all nonsingular matrices in $S_{n}(F)$. We only need to prove that $\operatorname{span} W=S_{n}(F)$, or to show that $E(i, i), D(i, j) \in \operatorname{span} W$ for all $i, j=1, \ldots, n, i<j$. 
For any $j \geq 2$, it is easily checked that the matrix $D(1, j)+I_{n}+E(j, j)$ is nonsingular. The same is also true for the matrix $D(1, j)+I_{n}+k E(1,1)$, where $k$ denotes the $k$-fold sum of the identity element of $F$, provided that $\operatorname{ch} F \not k$. Note that we have

$I_{n}+\sum_{j=2}^{n}\left\{\left[D(1, j)+I_{n}+k E(1,1)\right]-\left[D(1, j)+I_{n}+E(j, j)\right]\right\}=(k(n-1)+1) E(1,1)$.

If $\operatorname{ch} F \nmid n$, take $k=1$. Then $n E(1,1) \neq 0$ and $\operatorname{ch} F \nmid k$ (so that $D(1, j)+I_{n}+k E(1,1)$ is nonsingular); hence $E(1,1) \in \operatorname{span} W$. If $\operatorname{ch} F \mid n$ but $\operatorname{ch} F \neq 2$, take $k=2$. Then we have $\operatorname{ch} F \nmid k$ and also $E(1,1) \in \operatorname{span} W$. If $\operatorname{ch} F=2$, then

$$
E(1,1)=\left[E(1,1)+D(1,2)+\sum_{j=3}^{n} E(j, j)\right]+\left[D(1,2)+\sum_{j=3}^{n} E(j, j)\right]
$$

so we still have $E(1,1) \in \operatorname{span} W$. This proves that in all cases $E(1,1) \in \operatorname{span} W$. By symmetry, we also have $E(j, j) \in \operatorname{span} W$ for $j=2, \ldots, n$.

In view of the relation

$$
D(1,2)=\left(\left[\begin{array}{ll}
0 & 1 \\
1 & 0
\end{array}\right] \oplus I_{n-2}\right)-\sum_{j=3}^{n} E(j, j),
$$

and the proved fact that $E(j, j) \in \operatorname{span} W$ for all $j$, we have, $D(1,2) \in \operatorname{span} W$. By symmetry, we also have $D(i, j) \in \operatorname{span} W$ for all pairs of $i, j(i<j)$. The proof is completed. $\square$

Proof of Theorem 1.1: It is clear that we need only treat the "only if" part. We treat the case $\operatorname{ch} \mathbf{F} \neq 2$ first.

When $\operatorname{ch} \mathbf{F} \neq 2$, by the result of Lemma 2.5, it suffices to prove that $\phi$ is a linear transformation. Here we are borrowing (and correcting) the arguments used in the proof of [4, Proposition 2.1]. Note that condition (2) implies that for any $A, B \in S_{n}(\mathbf{F})$, the polynomial functions defined by the polynomials $D_{A, B}(t)$ and $D_{\phi(A), \phi(B)}(t)$ are equal. We want to prove that they are equal as polynomials. (In the proof of [4, Proposition 2.1], the latter is taken for granted.) In view of condition $(2), \phi$ preserves the determinant; so the coefficients of $t^{n}$ in the polynomials $D_{A, B}(t)$ and $D_{\phi(A), \phi(B)}(t)$, which are, respectively, $\operatorname{det} B$ and $\operatorname{det} \phi(B)$, are the same. Hence, the polynomial $D_{A, B}(t)-D_{\phi(A), \phi(B)}(t)$ is of degree at most $n-1$. On the other hand, by condition (2) again, each element of $F$ is a root of the latter polynomial. But $|\mathbf{F}| \geq n$, so the latter polynomial is equal to the zero polynomial. (The preceding argument also shows that in the hypothesis of [4, Proposition 2.1], we need to add the assumption that $|\mathbf{F}| \geq n$.) Let $\phi(A)=\left(\widetilde{a}_{i j}\right)$, by considering the coefficient of $t^{n-1}$, we have

$$
\sum_{i, j=1}^{n} a_{i j} B^{i j}=\sum_{i, j=1}^{n} \widetilde{a}_{i j} \phi(B)^{i j}
$$


Noting that $A$ and $B$ are symmetric and recalling that adj $B=\left(B^{j i}\right)$, we can rewrite the above as

$$
v_{\text {adj } B}^{t} \cdot \Gamma \cdot v_{A}=v_{\text {adj } \phi(B)}^{t} \cdot \Gamma \cdot v_{\phi(A)},
$$

where $\Gamma=\operatorname{diag}\left(1,2 I_{n-1}, 1,2 I_{n-2}, \cdots, 1,2 I_{2}, 1,2,1\right)$. Since $\operatorname{ch} \mathbf{F} \neq 2, \Gamma \in G L_{\frac{n(n+1)}{2}}(\mathbf{F})$.

By Lemma 2.6 there exists a basis for $S_{n}(F)$ consisting of nonsingular matrices, say $\left\{C_{1}, C_{2}, \ldots, C_{\frac{n(n+1)}{2}}\right\}$. Then for each $k$, adj $C_{k}=\left(\operatorname{det} C_{k}\right) C_{k}^{-1}$ and a simple calculation yields adj $\left(\operatorname{adj} C_{k}\right)=d_{k} C_{k}$, where $d_{k}=\left(\operatorname{det} C_{k}\right)^{n-2}$. For all $A \in S_{n}(F)$, by taking in (2.1) $B=\operatorname{adj} C_{k}$ for $k=1, \cdots, \frac{n(n+1)}{2}$, we obtain

$$
d_{k} v_{C_{k}}^{t} \cdot \Gamma \cdot v_{A}=v_{\text {adj } \phi\left(\operatorname{adj} C_{k}\right)}^{t} \cdot \Gamma \cdot v_{\phi(A)}, \text { for } k=1, \ldots, \frac{n(n+1)}{2} .
$$

Let $D, X$ and $Y$ be the matrices in $M_{\frac{n(n+1)}{2}}(\mathbf{F})$ given by

$$
D=\operatorname{diag}\left(d_{1}, \cdots, d_{\frac{n(n+1)}{2}}\right), \quad X=\left[\begin{array}{c}
v_{C_{1}}^{t} \\
v_{C_{2}}^{t} \\
\vdots \\
v_{C_{\frac{n(n+1)}{2}}^{t}}^{t}
\end{array}\right], \quad Y=\left[\begin{array}{c}
v_{\operatorname{adj} \phi\left(\operatorname{adj} C_{1}\right)}^{t} \\
v_{\operatorname{adj} \phi\left(\operatorname{adj} C_{2}\right)} \\
\vdots \\
v_{\operatorname{adj} \phi\left(\operatorname{adj} C_{\frac{n(n+1)}{2}}\right.}^{t}
\end{array}\right]
$$

Then we have

$$
D \cdot X \cdot \Gamma \cdot v_{A}=Y \cdot \Gamma \cdot v_{\phi(A)}, \text { for all } A \in S_{n}(\mathbf{F}) .
$$

It is clear that $v_{C_{1}}^{t}, \cdots, v_{\frac{n(n+1)}{2}}^{t}$ are linearly independent, as $C_{1}, C_{2}, \cdots, C_{\frac{n(n+1)}{2}}$ form a basis for $S_{n}(\mathbf{F})$. So $X$ is nonsingular. By taking in $(2.2) A=D(i, j)$ or $E(i, i)$ for all $i, j=1, \cdots, n, i<j$, we readily obtain

$$
D \cdot X \cdot \Gamma \cdot I_{\frac{n(n+1)}{2}}=Y \cdot \Gamma \cdot J
$$

where $J$ is the $\frac{n(n+1)}{2} \times \frac{n(n+1)}{2}$ matrix with columns $v_{\phi(D(i, j))}$ or $v_{\phi(E(i, j))}$ for $1 \leq i<$ $j \leq n$ arranged in the natural way. So $Y$ is nonsingular. By (2.2) we have

$$
\Gamma^{-1} Y^{-1} D X \Gamma v_{A}=v_{\phi(A)} \text { for all } A \in S_{n}(\mathbf{F}),
$$

which implies that the transformation $\phi_{1}$ from $\mathbf{F}^{\frac{n(n+1)}{2}}$ to itself given by

$$
\phi_{1}\left(v_{A}\right)=v_{\phi(A)} \text { for all } v_{A} \in \mathbf{F}^{\frac{n(n+1)}{2}},
$$

is linear. But $\phi=\sigma^{-1} \phi_{1} \sigma$, so $\phi$ is a linear transformation.

Now we treat the "only if" part for the case $\mathbf{F}=\mathbf{F}_{2}$ and $n=2$.

Let $\Gamma_{1}=S_{2}\left(\mathbf{F}_{2}\right) \backslash G L_{2}\left(\mathbf{F}_{2}\right)$ and $\Gamma_{2}=S_{2}\left(\mathbf{F}_{2}\right) \cap G L_{2}\left(\mathbf{F}_{2}\right)$. Then we have

$$
\begin{aligned}
& \Gamma_{1}=\left\{0,\left[\begin{array}{ll}
1 & 0 \\
0 & 0
\end{array}\right],\left[\begin{array}{ll}
0 & 0 \\
0 & 1
\end{array}\right],\left[\begin{array}{ll}
1 & 1 \\
1 & 1
\end{array}\right]\right\}, \\
& \Gamma_{2}=\left\{I_{2},\left[\begin{array}{ll}
1 & 1 \\
1 & 0
\end{array}\right],\left[\begin{array}{ll}
0 & 1 \\
1 & 1
\end{array}\right],\left[\begin{array}{ll}
0 & 1 \\
1 & 0
\end{array}\right]\right\} .
\end{aligned}
$$


We first show that $\phi(0)=0, \phi\left(\left[\begin{array}{ll}0 & 1 \\ 1 & 0\end{array}\right]\right)=\left[\begin{array}{ll}0 & 1 \\ 1 & 0\end{array}\right], \phi\left(\Gamma_{1} \backslash\{0\}\right)=\Gamma_{1} \backslash\{0\}$ and $\phi\left(\Gamma_{2} \backslash\left\{\left[\begin{array}{ll}0 & 1 \\ 1 & 0\end{array}\right]\right\}\right)=\Gamma_{2} \backslash\left\{\left[\begin{array}{ll}0 & 1 \\ 1 & 0\end{array}\right]\right\}$.

Since $\phi$ preserves the determinant, we have $\phi\left(\Gamma_{i}\right) \subseteq \Gamma_{i}$ for $i=1,2$. We can readily verify the following:

$1^{0} \operatorname{det}(A+B) \neq 0$ for every pair of distinct matrices $A, B \in \Gamma_{1} \backslash\{0\}$;

$2^{0} \operatorname{det}(0+A)=0$ for every $A \in \Gamma_{1} \backslash\{0\}$.

Since $\phi\left(\Gamma_{1}\right)=\Gamma_{1}$, by $1^{0}, 2^{0}$ and condition (2) it follows that we have $\phi\left(\Gamma_{1} \backslash\{0\}\right)=$ $\Gamma_{1} \backslash\{0\}$ and $\phi(0)=0$.

Similarly, by making use of the fact that $\phi\left(\Gamma_{2}\right) \subseteq \Gamma_{2}$ and that $\operatorname{det}(A+B) \neq 0$ for every pair of distinct matrices $A, B \in \Gamma_{2} \backslash\left\{\left[\begin{array}{ll}0 & 1 \\ 1 & 0\end{array}\right]\right\}$, we can also show that $\phi\left(\Gamma_{2} \backslash\left\{\left[\begin{array}{ll}0 & 1 \\ 1 & 0\end{array}\right]\right\}\right)=\Gamma_{2} \backslash\left\{\left[\begin{array}{ll}0 & 1 \\ 1 & 0\end{array}\right]\right\}$ and $\phi\left(\left[\begin{array}{ll}0 & 1 \\ 1 & 0\end{array}\right]\right)=\left[\begin{array}{ll}0 & 1 \\ 1 & 0\end{array}\right]$.

¿From the above discussion, $\phi\left(I_{2}\right)$ equals $I_{2},\left[\begin{array}{ll}1 & 1 \\ 1 & 0\end{array}\right]$ or $\left[\begin{array}{ll}0 & 1 \\ 1 & 1\end{array}\right]$. Consider the case when $\phi\left(I_{2}\right)=I_{2}$. Then $\phi\left(\left[\begin{array}{ll}1 & 1 \\ 1 & 0\end{array}\right]\right)$ equals $\left[\begin{array}{ll}1 & 1 \\ 1 & 0\end{array}\right]$ or $\left[\begin{array}{ll}0 & 1 \\ 1 & 1\end{array}\right]$. Suppose the former happens. Then $\phi(A)=A$ for all $A \in \Gamma_{2}$ and we can show that $\phi$ is in fact the identity transformation of $S_{2}(F)$ as follows.

Note that the sum of $\left[\begin{array}{ll}1 & 1 \\ 1 & 1\end{array}\right]$ and $I_{2}$ belongs to $\Gamma_{2}$, whereas the sum of $I_{2}$ and $\left[\begin{array}{ll}1 & 0 \\ 0 & 0\end{array}\right]$ (or $\left[\begin{array}{ll}0 & 0 \\ 0 & 1\end{array}\right]$ ) belongs to $\Gamma_{1}$. But $\phi$ fixes $I_{2}$, it follows that $\phi$ also fixes $\left[\begin{array}{ll}1 & 1 \\ 1 & 1\end{array}\right]$. By a similar argument, we can also show that $\phi$ fixes $\left[\begin{array}{ll}1 & 0 \\ 0 & 0\end{array}\right]$ and $\left[\begin{array}{ll}0 & 0 \\ 0 & 1\end{array}\right]$. So $\phi$ is the identity transformation when $\phi\left(I_{2}\right)=I_{2}$ and $\phi\left(\left[\begin{array}{ll}1 & 1 \\ 1 & 0\end{array}\right]\right)=$ $\left[\begin{array}{ll}1 & 1 \\ 1 & 0\end{array}\right]$

$$
\begin{gathered}
\text { If } \phi\left(I_{2}\right)=I_{2} \text { and } \phi\left(\left[\begin{array}{ll}
1 & 1 \\
1 & 0
\end{array}\right]\right)=\left[\begin{array}{ll}
0 & 1 \\
1 & 1
\end{array}\right] \text {, then necessarily } \\
\phi\left(\left[\begin{array}{ll}
0 & 1 \\
1 & 1
\end{array}\right]\right)=\left[\begin{array}{ll}
1 & 1 \\
1 & 0
\end{array}\right] .
\end{gathered}
$$

Using the same kind of argument as before, we can then show that

$$
\begin{aligned}
& \phi\left(\left[\begin{array}{ll}
1 & 1 \\
1 & 1
\end{array}\right]\right)=\left[\begin{array}{ll}
1 & 1 \\
1 & 1
\end{array}\right], \\
& \phi\left(\left[\begin{array}{ll}
1 & 0 \\
0 & 0
\end{array}\right]\right)=\left[\begin{array}{ll}
0 & 0 \\
0 & 1
\end{array}\right]
\end{aligned}
$$


and

$$
\phi\left(\left[\begin{array}{ll}
0 & 0 \\
0 & 1
\end{array}\right]\right)=\left[\begin{array}{ll}
1 & 0 \\
0 & 0
\end{array}\right] .
$$

The preceding argument shows that there are at most two transformations $\phi$ that satisfy $\phi\left(I_{2}\right)=I_{2}$ and condition (2). Likewise, we can also show that there are at most two transformations $\phi$ that satisfy condition $(2)$ for which $\phi\left(I_{2}\right)=\left[\begin{array}{ll}1 & 1 \\ 1 & 0\end{array}\right]$ (or $\left.\left[\begin{array}{ll}0 & 1 \\ 1 & 1\end{array}\right]\right)$. So altogether there are at most six transformations $\varphi: S_{2}\left(\mathbf{F}_{2}\right) \rightarrow S_{2}\left(\mathbf{F}_{2}\right)$ that satisfy condition (2). On the other hand, there are precisely six matrices in $G L_{2}\left(F_{2}\right)$, namely, $\left[\begin{array}{ll}1 & 0 \\ 0 & 1\end{array}\right],\left[\begin{array}{ll}1 & 1 \\ 0 & 1\end{array}\right],\left[\begin{array}{ll}1 & 0 \\ 1 & 1\end{array}\right],\left[\begin{array}{ll}0 & 1 \\ 1 & 0\end{array}\right],\left[\begin{array}{ll}1 & 1 \\ 1 & 0\end{array}\right]$ and $\left[\begin{array}{ll}0 & 1 \\ 1 & 1\end{array}\right]$. For each such matrix $P$ the transformation $\phi$ given by $\phi(A)=P A P^{t}$ clearly satisfies condition (2), and also it is readily checked that the six transformations arising in this way are different. This proves our result.

We do not know whether Theorem 1.1 can be extended to include the cases $\operatorname{ch} \mathbf{F}=2$ (but not $\mathbf{F}=\mathbf{F}_{2}$ and $n=2$, which is already covered), and $|\mathbf{F}|=3$ with $n=2$ or 3 .

Acknowledgment. The authors would like to thank the referee for his invaluable comments on and suggestions to an earlier version of the paper. We express our gratitude to Professor Bit-Shun Tam for many useful discussions and helps concerning the matter of this paper.

\section{REFERENCES}

[1] G. Frobenius. Über die Darstellung der endlichen Gruppen durch lineare Substitutionen. S. B. Preuss. Akad. Wiss., Berlin, pp. 994-1015, 1897.

[2] G. Dolinar and P. S Semrl. Determinant preserving maps on matrix algebras. Linear Algebra Appl., 348:189-192, 2002.

[3] N. Jacobson. Lectures in abstract algebra, Vol II. reprint of the 1953 edition [Van Nostrand, Toronto], Graduate Texts in Mathematics No. 31, Springer-Verlag, New York-Berlin, 1975.

[4] V. Tan and F. Wang. On determinant preserver problems. Linear Algebra Appl., 369:311-317, 2003.

[5] S.W. Liu and J.J. Guo. Rank one preservers on symmetric matrix spaces. J. of Natural Sci. Heilongjiang Univ., 16(1):5-8, 1999. 